\title{
The Motility of Human Milk Macrophages in Collagen Gels ${ }^{1}$
}

\author{
FATIH ÖZKARAGÖZ, H. BETH RUDLOFF, SRINIVASAN RAJARAMAN, AKRAM A. MUSHTAHA, \\ FRANK C. SCHMALSTIEG, AND ARMOND S. GOLDMAN
}

Departments of Pediatrics, Pathology, and Human Biological Chemistry and Genetics, The University of Texas

Medical Branch, Galveston, Texas 77550

\begin{abstract}
The hypothesis that the diminished motility of human milk leukocytes is due to a decrease in adherence was tested by using a collagen gel system in which leukocyte movement is less dependent on adherence. Unfractionated human milk leukocytes (HML) or fractionated peripheral blood leukocytes were placed on collagen gels in microwells and the leading edge of migration was determined by inverted phase microscopy. The mean rates of invasion of HML, blood neutrophils, and mononuclear blood leukocytes were 14,240 , and $<1 \mu / \mathrm{h}$, respectively $(p<0.01)$. We then examined the identity of motile HML by immunoperoxidase techniques using antibodies to selected cell markers. Motile HML were positive for a specific macrophage marker (cathepsin B) and a neutrophil and monocyte marker (Mac-1) but were negative for specific neutrophil (cathepsin G) or lymphocyte markers (CD3 and CD5). The directed motility of these cells was not enhanced by exposure to the chemoattractant, $\mathrm{N}$-formylL-methionyl-L-phenylalanine, but was inhibited by a $\mathrm{T}$ cell lectin, phytohemagglutinin. The movement was actin dependent but was not dependent on calcium or Mac-1 surface glycoproteins. Thus, the diminished motility of milk neutrophils does not appear to be due to decreased adherence per se, and those HML that are motile are macrophages. This suggests a dichotomy for the function of HML. Neutrophils may be relegated to the lumen of the alimentary tract, whereas macrophages may penetrate into mucosal sites for host defense. (Pediatr Res 23: 449-452, 1988)
\end{abstract}

\section{Abbreviations}

HML, human milk leukocyte(s)

PBL, peripheral blood leukocyte(s)

F-Met-Phe, N-formyl-L-methionyl-L-phenylalanine

PHA, phytohemagglutinin

Recent investigations have demonstrated that the motility of neutrophils in human milk is greatly decreased (1-3), and that their adherence, orientation, or directed movements are not increased after exposure to chemoattractant agents (3). In one study (3), preliminary evidence was found that some mononuclear cells in human milk moved spontaneously in a two-dimensional system, e.g. under agarose. Those findings were in keeping

Received October 5, 1987; accepted December 23, 1987. Correspondence Armond S. Goldman, M.D., Department of Pediatrics, Child Health Center C2-31, University of Texas Medical Branch, Galveston, TX 77550.

Supported in part by contracts from the National Institute of Child Health Development (HD 21049-01) and the U.S. Department of Agriculture (58-7MNI6-100).

${ }^{1}$ Presented at the 1987 meeting of the Society for Pediatric Research. with earlier observations that lymphocytes and macrophages from human milk could migrate on glass (4-6).

Herein, we extended the previous investigations by posing two new questions. The first was whether the decreased motility of human milk neutrophils was incidental to defective adherence. The design of those experiments was based on the fact that motility in two-dimensional systems involves adherence-dependent, traction events, whereas movement in three-dimensional models such as collagen is largely adherence-independent and involves hydraulic or pushing motions (7). We thus exploited this difference to examine the adherence-independent motility of HML in a type I collagen gel matrix. These experiments also allowed us to address the second question, the identity of the types of motile mononuclear HML and to subsequently quantitate the movement of those cells, to determine whether their movement was dependent on surface glycoproteins that mediate cellular adherence or on actin filaments that are necessary for the propulsion of other cells, and to ascertain whether their movement could be modulated by other agents.

\section{METHODS}

Donor selection and specimen preparations. The study was approved by the Institutional Review Board for Human Research. Venous blood was collected from healthy adult volunteers. PBL were subjected to sedimentation with $6 \%$ dextran and then to Ficoll-Hypaque (Sigma Chemical Co., St. Louis, MO) density gradients (7) to obtain neutrophils ( $>95 \%$ pure) and mononuclear cells ( $75 \%$ pure). Unfractionated HML were prepared in the following manner. Human colostrum collected from healthy women in the first 3 days after delivery (8) was diluted 1:4 in RPMI-1640 (Whittaker M.A. Bioproducts, Walkersville, MD) and centrifuged at $100 \times g$ for $5 \mathrm{~min}$. The cellular pellets were then resuspended in RPMI 1640 to achieve a final concentration of $1 \times 10^{7}$ leukocytes $/ \mathrm{ml}$. Viability of the cells was determined by trypan blue exclusion. Macrophages were detected by the presence of nonspecific esterase and neutrophils by myeloperoxidase (10). The relative frequencies of HML in the preparations (neutrophils 40 to $60 \%$; macrophages 35 to $55 \%$; lymphocytes 5 to $8 \%$ ) were similar to that found in previously (3).

Preparation of collagen gels. Type I collagen was prepared from stripped rat tendons that were solubilized in cold $3 \%(\mathrm{v} / \mathrm{v})$ acetic acid for 2 days (11). Clear supernatant preparations obtained by centrifugation were mixed with $20 \%(\mathrm{w} / \mathrm{v}) \mathrm{NaCl}$. The precipitated type I collagen was pelleted by centrifugation at $3000 \times g$ for $1 \mathrm{~h}$, washed and dissolved in $3 \%$ acetic acid (final concentration $1.2 \mathrm{mg} / \mathrm{ml}$ ). The solution was dialyzed against distilled $\mathrm{H}_{2} \mathrm{O}$ that was adjusted to $\mathrm{pH} 4.0$ with $\mathrm{HCl}$. Eight ml of the collagen solution was mixed with $1 \mathrm{ml}$ of a $4 \%(\mathrm{w} / \mathrm{v})$ solution of sodium bicarbonate and $1 \mathrm{ml}$ of a 10 -fold concentrated solution of minimal essential medium (Eagle modified, McLean Flow Laboratories, Inc., McLean, VA) to restore the $\mathrm{pH}$ to 7.4 
and the ionic strength to physiologic levels. In certain experiments, one of the following was added to some collagen preparations before gelling: decomplemented or fresh human serum $(10 \%)$, acellular human colostrum $(10 \%)$, monoclonal antibodies to Mac-1 or its $\beta$-subunit (13), or F-Met-Phe (Sigma) (40 $\mu \mathrm{g} \mathrm{F-}$ Met-Phe/ml of collagen solution).

Cellular penetration into collagen gels. Unstimulated movement was tested in the following manner. A total of $50 \mu \mathrm{l}$ of a fresh solution of collagen was placed in each well of flat bottom, 96-well microtiter plates (Becton Dickinson, Oxnard, CA) and allowed to gel by incubating the plates at $37^{\circ} \mathrm{C}$ for $15 \mathrm{~min}$. Unfractionated HML or fractionated PBL $\left(2 \times 10^{5}\right.$ cells $/ 25 \mu l$ RPMI 1640) were placed on the surface of each gel and were then incubated at $37^{\circ} \mathrm{C}$. The leading edge of migrating cells was determined at 1-24 h by direct microscopy (12) (Diavert, Inverted Phase Microscope, Leitz, West Germany).

Phenotypic characterization of motile human milk leukocytes. In these experiments the types of HML that invaded the collagen gels were ascertained after $24 \mathrm{~h}$ of incubation. Gels were stained for myeloperoxidase (10) to identify neutrophils and for nonspecific esterase (10) to identify macrophages and then examined microscopically. Immunocytologic examinations were carried out in the following manner. Gels were quick-frozen in precooled isopentane and embedded in O.C.T. Compound (Tissue-Tek Miles Scientific, Naperville, IL). After the top portion of the gel was discarded, it was vertically sliced into serial $4-\mu$ sections. Sections were air dried for $60 \mathrm{~min}$ and fixed in cold acetone $\left(-20^{\circ} \mathrm{C}\right)$ for $10 \mathrm{~min}$. After rehydration, they were incubated with the following primary antibodies: sheep anticathepsin B (14) and anticathepsin G (15) (ICN Immunobiologicals, Lisle, IL), and mouse monoclonal anti-OKT3 (CD3), anti-Leu 1 (CD5), antiLeu7 (Becton Dickinson, Mountainview, CA), $(16,17)$ (Ortho Diagnostic System Inc., Raritan, NJ), and anti-Mac-1 (prepared in our laboratory). The preparations were then exposed accordingly to either biotinylated rabbit anti-sheep IgG (Vector Labs., Burkingame, CA) or biotinylated sheep anti-mouse IgG (Cooper Biomedicals, Westchester, PA) (1:80). Finally, the reaction with Streptavidin biotinylated peroxidase complex (Amersham. Arlington Heights, IL) (1:500) was visualized by incubating with diaminobenzadine and hydrogen peroxide.

Modulation of the motility of human milk leukocytes. Certain requirements for the motility of HML and the effects of certain modulators on these cells were tested. The need for actin filament was examined by exposing these cells to $1 \mathrm{mM}$ cytochalasin $\mathrm{B}$ (Sigma). The necessity for calcium was tested by treating the cells with a $10 \mathrm{mM}$ solution of EDTA (Sigma) for $20 \mathrm{~min}$ before they were placed on collagen. Inasmuch as human milk macrophages display Mac-1 on their surfaces (unpublished data), the need for this protein for motility in this system was assessed by reacting the cells with mouse monoclonal antibodies to Mac-1 prepared in our laboratory or with monoclonal antibodies to its $\beta$-subunit (13) for $20 \mathrm{~min}$ before their movement was tested and by adding the same monoclonal antibodies to the collagen solution before gelling. The possible inhibitory effect of serum was examined by incubating $\mathrm{HML}$ in $10 \%$ fresh human serum overnight at $21^{\circ} \mathrm{C}$ on a horizontal shaker. The influence of PHA (Difco Corp., Detroit, MI) on HML was tested with concentrations of PHA ranging from 10 times to 0.001 of the optimal mitogenic dose (20 $\mu \mathrm{l} / 100 \mu \mathrm{l}$ RPMI 1640). The effects of F-Met-Phe on the motility of colostral cells were determined by employing collagen gels prepared with F-Met-Phe as described above. Controls for these experiments consisted of aliquots of untreated cells placed on unmodified collagen gels.

Effects of acellular human colostrum. The effects of acellular human colostrum on the motility of human blood mononuclear leukocytes were measured by incubating them in previously stored $\left(-80^{\circ} \mathrm{C}\right.$ ) acellular milk $(10 \%)(3)$ for $15 \mathrm{~min}$. Motility was determined $4 \mathrm{~h}$ after the cells were applied to the gel. Controls for these experiments were peripheral blood mononuclear leukocytes incubated in RPMI 1640.
Statistical analysis. Data concerning the leading edge of cell migration were expressed as the means and $95 \%$ confidence intervals. Differences between the degree of movement of HML and neutrophils or mononuclear leukocytes in human blood were tested by an unpaired Student's $t$ test, whereas the effects of modulating agents (human serum, acellular human colostrum, cytochalasin B, EDTA, anti-Mac- 1 , anti- $\beta$-subunit, PHA, or FMet-Phe) on leukocytes were tested by a paired Student's $t$ test.

\section{RESULTS}

Invasion of leukocytes into collagen gels. As previously reported (18), peripheral blood neutrophils rapidly invaded the collagen gels $(240 \mu / \mathrm{h})$, whereas few peripheral blood mononuclear cells penetrated the gels during the period of observation (Fig. 1). The rate of penetration of HML into the gels $(13 \mu / \mathrm{h})$ was much slower than peripheral blood neutrophils $(p<0.001)$, but more rapid than the blood mononuclear cells $(p<0.001)$ (Fig. 1).

Identification of motile HML. The identity of motile HML was sought in specimens obtained from six individuals. In each case, although neutrophils and lymphocytes as well as macrophages were found on the surface of the gels, the only HML which invaded the collagen matrix displayed markers for macrophages (nonspecific esterase, cathepsin B), but not for neutrophils or lymphocytes (Table 1).

Modulation of motility. Cytochalasin B inhibited the migration of human milk macrophages $(p<0.001)$, whereas the rate of migration of EDTA-, anti-Mac-1-, or anti- $\beta$-subunit-treated HML were similar to the rate of spontaneous motility of untreated cells $(15.5,13.8,14$, and $13 \mu / \mathrm{h}$, respectively) (Fig. 2). The motility of human milk macrophages was reduced significantly after exposure to PHA $(p<0.001)$. Also the inhibition was directly proportional to the amount of PHA that was used (range 0.01-10 times the optimal mitogenic dose; data not shown). The rates of migration of HML in control and F-MetPhe-stimulated preparations were similar $(13.0$ and $12.5 \mu / \mathrm{h}$, respectively) (Fig. 2). Finally, the motility of peripheral blood mononuclear cells was unaffected by acellular human colostrum $(<1 \mu / \mathrm{h}, p>0.05)$ and the motility of HML was not affected by human serum $(12.0 \mu / \mathrm{h}, p>0.05$; data not shown).

\section{DISCUSSION}

The results of these experiments and previous studies (1-4) indicate that the types of human milk leukocytes that migrate spontaneously or in response to stimulating agents are quite different from those of peripheral blood. Blood neutrophils display spontaneous movement in in vitro systems and their movements are enhanced by chemoattractant agents $(19,20)$. In contrast, neutrophils from human milk do not move spontaneously or in response to chemoattractants in either two-dimensional $(1-3)$ or, as in the present study, three-dimensional systems. The cause of the decrease in motility of neotrophils from human milk is unclear. We previously reported that the motility of peripheral blood neutrophils in a two dimensional system was not decreased by incubating them in human colostrum (3). In addition, the rates of movement of blood neutrophils obtained early in lactation and from nonpregnant, nonlactating adults were indistinguishable. Because movement into collagen gels by human blood neutrophils does not appear to be dependent on adherence (18), the failure of human milk neutrophils to invade the matrix may be due to a different mechanism such as a lack of deformability as previously suggested (3).

Motile leukocytes in human milk, as shown by cytochemical and immunochemical markers, proved to be macrophages. The rate of movement of these cells was less than peripheral blood neutrophils, but much greater than blood mononuclear cells. In these studies, we found that their motility was actin-dependent as demonstrated by the response to an inhibitor of actin polym- 


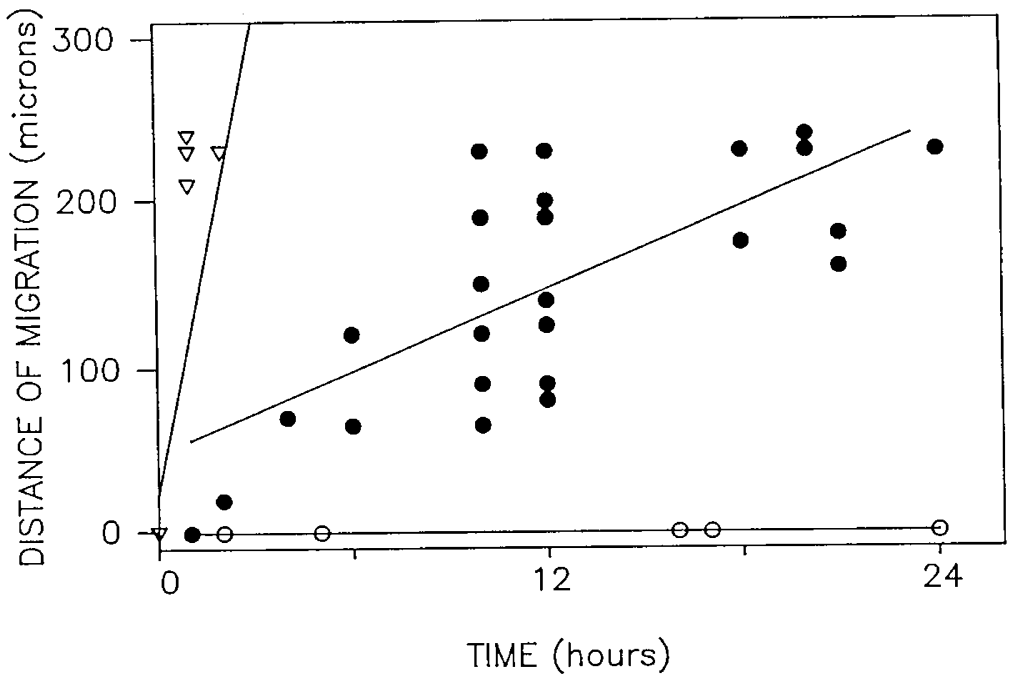

Fig. 1. The rates of invasion of peripheral blood neutrophils $(\nabla), \operatorname{HML}(\bullet)$, and blood mononuclear leukocytes $(O)$ into collagen gels. Data from 28 experiments are displayed, but not all data points are shown because of overlapping values. First order regression lines are also included. The rates of movement of HML were slower than peripheral blood neutrophils $(p<0.001)$, but greater than blood mononuclear leukocytes $(p<0.001)$.

Table 1. Immunologic-cytochemical markers of motile HML invading collagen gels

\begin{tabular}{lcccc}
\hline & \multicolumn{4}{c}{ Type of leukocytes } \\
\cline { 2 - 5 } \multicolumn{1}{c}{ Cell markers } & $\begin{array}{c}\text { Motile } \\
\text { HML }\end{array}$ & $\begin{array}{c}\text { Monocytes } \\
\text { macrophages }\end{array}$ & $\begin{array}{c}\text { Neutro- } \\
\text { phils }\end{array}$ & $\begin{array}{c}\text { Lympho- } \\
\text { cytes }\end{array}$ \\
\hline Nonspecific esterase & + & + & - & - \\
Cathepsin B & + & + & - & - \\
Mac-1 & + & + & + & - \\
Myeloperoxidase & - & - & + & - \\
Cathepsin G & - & - & + & - \\
CD5, CD3, Leu7 & - & - & - & + \\
\hline
\end{tabular}

erization (cytochalasin B), but was calcium-independent since a calcium chelator (EDTA) did not alter their motility. Furthermore, the motility of human milk macrophages in the collagen matrix was found to be similar to blood neutrophils in that it was not dependent upon adherence mediated by Mac- 1 or its $\beta$ subunit. Finally, it is of interest that the motility of milk macrophages was greatly inhibited by PHA. Although the genesis of this effect was not explored, it is possible that the inhibition was caused by production of lymphokines, such as macrophage migration inhibition factor from milk lymphocytes (21).

Our studies failed to elucidate why the motility of human milk macrophages is much greater than that of blood mononuclear cells because human serum did not inhibit the movement of milk macrophages and human colostrum did not augment the motility of blood mononuclear leukocytes. It is important to understand, however, that the in vitro conditions of these experiments, e.g. the duration of exposure, concentration of reagents, physiologic state of the cells, may not have been approximated in vivo conditions.

Regardless of the mechanisms that are responsible for the altered motility of HML, a dichotomy of function of HML based on the presence or absence of motility is suggested. The action of human milk neutrophils may be relegated to the lumen of the alimentary tract, whereas the macrophages may interact with the epithelium of the aeroalimentary tract of the recipient infant. It is not clear, however, how these cells would function in those circumstances.

Note added in proof. Since this paper was accepted, we have found that the motility of blood monocytes is enhanced by incubating them in human milk for $3 \mathrm{~h}$.

Acknowledgments. The authors gratefully acknowledge the

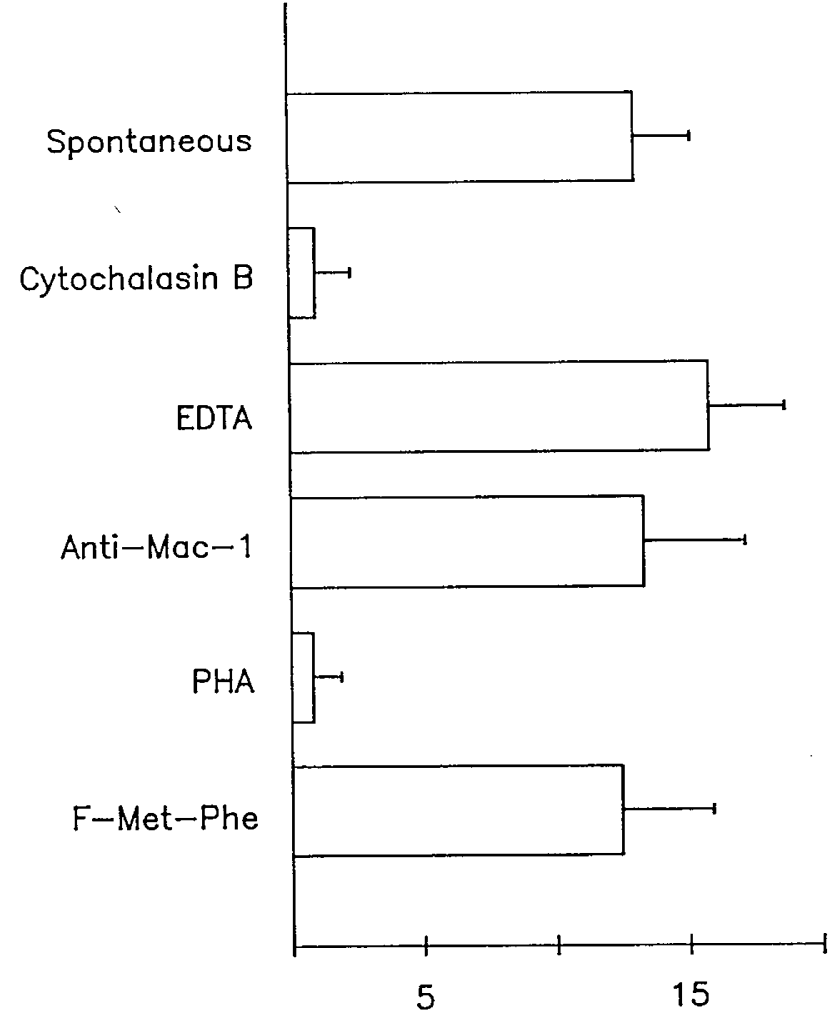

RATE OF MIGRATION (microns/h)

Fig. 2. The effect of modulating agents on the motility of human milk macrophages. Data are presented as the mean and $95 \%$ confidence interval of five to 25 observations for each experiment. Since there were no differences between the results of the anti-Mac-1 and the anti- $\beta$ subunit studies, only the results of the anti-Mac-1 experiments were presented. The rate of movement of the cells was significantly decreased after treatment with cytochalasin B $(p<0.001)$ or PHA $(p<0.001)$.

technical assistance of Kimberly Palkowetz and Debra Pyron and the secretarial aid of Pat Muldrow.

\section{REFERENCES}

1. Khan AJ, Rosenfeld W, Vadapalli M, Biagton J, Khan P, Hug A, Evans HE 1980 Chemotaxis and random migration of human milk cells. J Pediatr $96 ; 879-882$ 
2. Hawes CS, Jones WR 1985 Human milk cell migration and production of monocyte chemotactic factor: lack of activity. Pediatr Res 19:996-999

3. Thorpe LW, Rudloff HE, Powell LC, Goldman AS 1986 Decreased response of human milk leukocytes to chemoattractant peptides. Pediatr Res 20;373377

4. Smith CW, Goldman AS 1968 The cells of human colostrum. I. In vivo studies of morphology and function. Pediatr Res 2:103-109

5. Smith CW, Goldman AS 1970 Interactions of lymphocytes and macrophage from human colostrum: Characteristics of the interacting lymphocyte. J Reticuloendothel Soc 8:91-104

6. Smith CW, Goldman AS, Yates RD 1971 Interactions of lymphocytes and macrophages from human colostrum: electron microscopic studies of the interacting lymphocyte. Exp Cell Res 69:409-415

8. Böyum, A 1968 Isolation of leukocytes from human blood. Further observations. Methylcellulose, dextran and ficoll as erythrocyte-aggregating agents. Scand J Clin Lab Invest 21 (suppl 97):31-50

9. Goldblum RM, Garza C, Johnson CA, Nichols BL, Goldman AS 1981 Human milk banking. I. Effects of container upon immunologic factors in mature milk. Nutr Res 1:449-459

10. Yam LT, Li CY, Crosby WH 1970 Cytochemical identification of monocytes and granulocytes. AJCP 55:283-290

11. Schor SL, Allen TD, Harrison CJ 1980 Cell migration through three-dimensional gels of native collagen fibres: collagenase activity is not required for the migration of two permanent cell lines. J Cell Sci 46:171-186

12. Zigmond SH, Hirsch JG 1973 Leukocyte locomotion and chemotaxis: New methods for evaluation and demonstration of cell-derived chemotactic factor. J Exp Med 137:387-410
13. Sanchez-Madrid F, Nagy JA, Robbins E, Simon P, Springer TA 1983 A human leukocyte differentiation antigen family with distinct alpha subunits and a common beta subunit: the lymphocyte-function associated antigen (LFA-1), the C3bi complement receptor (OKMI/Mac-1), and the p150,95 molecule. J Exp Med 158:1785-98

14. Morland B 1985 Cathepsin B activity in human blood monocytes during differentiation in vitro. Scand J Immunol 22:9-16

15. Senior RM, Campbell EJ 1984 Cathepsin G in human mononuclear phagocytes: comparisons between monocytes and U937 monocyte-like cells. J Immunol 132:2547-2551

16. Jondal J, Wigzell H, Aiuti F 1973 Human lymphocyte subpopulations: Classification according to surface markers and/or functional characteristics. Transplant Rev 16:163-195

17. van Agthoven A, Terhorst C, Reinherz E, Schlossman S 1981 Characterization of T cell surface glycoproteins T11 and T3 present on all human peripheral T lymphocytes and functionally mature thymocytes. Eur J Immunol 11:1821

18. Schmalstieg FC, Rudloff HE, Hillman GR, Anderson DC 1986 Two dimen sional and three dimensional movement of human polymorphonuclear leukocytes: Two fundamentally different mechanisms of locomotion. J Leukocyte Biol 40;677-691

19. Ward PA, Cochrane CG, Müller-Eberhard HJ 1965 The role of serum complement in chemotaxis of leukocytes in vitro. J Exp Med 122:327-346

20. Schiffman E, Corcoran BA, Wahl SM $1975 \mathrm{~N}$-formylmethionyl peptides as chemoattractants for leukocytes. Proc Nat Acad Sci USA 72:1059-1062

21. Keller MA, Kidd RM, Bryson YJ, Turner JL, Carter J 1981 Lymphokine production by human milk lymphocytes. Infect Immun 32:632-636 